\title{
Research and design of intelligent image recognition mechanism and its application
}

\author{
Wang Xiaoyuan ${ }^{1, a}$, Wang Jianping ${ }^{2}$ and Wang Hongfei ${ }^{3}$ and Cheng Wenbing ${ }^{1}$ \\ ${ }^{1}$ Hefei University teaching and experimental center, 230022, Hefei, AnHui, China \\ ${ }^{2}$ College of electrical automation, HeFei University of Technology professor,230069,Hefei, AnHui,China \\ ${ }^{3}$ College of physics and electronics, Henan University 475001, Kaifeng, HeNan, China
}

\begin{abstract}
In this paper, an in-depth study on the recognition mechanism (identification experts) and behavioral function simulation is made, and a design method has been developed ----- Image recognition mechanism. The research results and methods are expounded. At the same time, experiments are carried out on the above design methods. The effectiveness and feasibility of this design have been proved by experiments. Furthermore, the design of the expert observer is carried out.The design of artificial intelligent character recognition machine. Its ability to recognize complex background images has a high success rate, which has been proved by experiments, and its adaptive ability is very strong.
\end{abstract}

\section{Introduction}

Machine image recognition is a branch of image recognition system, and people pay great attention to the accuracy of sub-graph location segmentation, especially the problem of object recognition. This article explores in depth the basic structure and function (EISR) required for the above problems:

- The structure level is divided into three levels: (abbreviated as Pattern Tuning), a recognition level (Recognition abbreviated as RE); mode correction level), that is, two levels of parallel coupling structure.

- Two levels of functions are required: state modeling and parameter characterization in online identification. On the basis of qualitative and quantitative analysis, parameter identification strategy and recognition and multi-mode state are presented.

- Data-driven reasoning as object features--Fuzzy reasoning in human-like (expert) decision-making process.

This paper argues that : the feature space ------ is the basis of intelligent recognition knowledge, qualitative description of the physical model of the system.According to the combination of artificial intelligence and image processing technology, pattern recognition theory and computer technology, the design method of expert recognition machine is established.

\section{Establish intelligent identification theory}

In this paper, the viewpoint and recognition mechanism of behavioral function simulation are studied, and several conditions are set as follows:

\subsection{Condition 1:}

Let: the chara cteristic model is a description of the space where the object is identified, namely the feature space, in the condition of intelligent identification system. It is also a division of information space $\sum$, which is corresponding to identified objects. For the specific requirements of this division is based on the identification of problems.

The characteristic state $\Phi_{i}$ is the region of each partition used to represent a state that identifies an object. The feature model is the general term of the set of all feature states. Can be expressed as:

$$
\Phi=\left\{\phi_{1}, \phi_{2}, \cdots, \phi_{i}\right\} \quad \phi_{i} \in \sum
$$

The state information space of the whole ----- $\Sigma$;

A combination of attributes or attributes that an object ------has $\phi_{i}$.

According to the above, make: the pattern class corresponds to the feature model. Pattern class ------ is determined based on problem identification requirements. Its essence is a kind of "homomorphism" model, which is specific to information space. As different problems require ------ to identify output, parameter correction, task adaptation, etc., the feature model of the same information space will be different.

The feature element ------ its combination is used to describe the feature state. To:The feature primitive set is expressed as:

$$
Q=\left\{q_{1}, q_{2}, \cdots q_{m}\right\}
$$

Characteristic state $\phi_{i}$------The set composed of feature primitives is described by qualitative and quantitative methods.

\footnotetext{
${ }^{a}$ Corresponding author: hfuu.wangxy@163.com
} 
The relation between feature model and feature primitive set is:

$\Phi=P \otimes Q$

$\mathrm{M}$ dimensional characteristic primitives vector------ $Q$;

The vector of n----- $\Phi$.

matrix of relation $\left.{ }^{P=\left[p_{i j}\right.}\right]_{n \times m}$.

$\otimes$ Denotes the multiplication relation of "and" matrix; $p_{i j}$ For $-1,0$, 1, each is negative, zero sum positive. That is:

$$
\Phi_{i}=\left[\left(p_{i 1} \bullet q_{1}\right) \cap\left(p_{i 2} \bullet q_{2}\right) \cap \cdots \cap\left(p_{i m} \bullet q_{m}\right)\right]
$$

\subsection{Condition 2:}

G ------ is the set and space of the pattern to be recognized. F ------ feature space.

The description of feature extraction is $\mathrm{f} \subseteq \mathrm{G} \times \mathrm{F}$, which is a two element relationship between $G$ and $F$. Among them, f ------ represents a general two element relationship between $F$ and $G$. It is expressed as specialized 1-N mapping and 1-1 mapping. If different patterns are used for feature extraction, multiple feature spaces are obtained. Let $\mathrm{k}$ be some positive integer, $\mathrm{F} 1, \mathrm{~F} 2 \ldots \mathrm{Fk}$, total $\mathrm{k}$, this process is characterized by feature extraction in the aspect of multi-lateral degrees, which can be respectively described.Use a binary relation $\mathrm{fi} \subseteq \mathrm{G} \times \mathrm{Fi}(\mathrm{i}=1,2 \ldots, \mathrm{k})$. In the case of multiple features, it is generally after the following situation, that is, after expansion into the multi-feature space duality relations. At this point, the focus of the research ------ to facilitate classification, the integration of multiple characteristics. In the case of the result of extraction without loss of information, the characteristics of the fusion are discussed. Therefore, the recognition information is not lost, which is to achieve a purpose of joint or associative recognition, and more importantly, whether the recognition characteristics of the pattern can be comprehensively reflected, which is used in multilateral feature extraction.

\subsection{Condition 3:}

Feature identification------ is carried out in the case of SHIR according to the feature model, while processing the feature extraction information. It is also called pattern recognition. At the same time, it recognizes the object in the process of characteristic state.

\subsection{Condition 4:}

Feature Memory - Memory of the feature information of the standard pattern, is a concept used for humanoid intelligent recognizer. It is the effect of early recognition and decision-making based on expert knowledge, and it is the reflection of the requirement of recognition object and the nature of recognition task.

The set of characteristic memory quantities is expressed as:

$$
\wedge=\left\{\lambda_{1}, \lambda_{2}, \ldots, \lambda_{p}\right\}, \quad \lambda_{i} \in \sum
$$

Feature memory ------ basic basis for feature discrimination, which directly affects the adjustment of output and recognition, also includes the following aspects: self-adaptation, self-correction and self-learning.

\subsection{Condition 5:}

The set of Decision Model $\Psi$------ is the input information $U$ in the feature memory information, the input information $\mathrm{E}$ of the recognition machine and the feature memory information $\wedge$ (collectively referred to as $\mathrm{R}$ ), having some kind of quantitative or qualitative mapping relationship------The set of F. Can be expressed as:

$$
\begin{aligned}
& \Psi=\left\{\Psi_{1}, \Psi_{2}, \ldots, \Psi_{r}\right\} \\
& \Psi_{i}: u_{i}=j_{i}\left(x_{1}, x_{2}, \ldots, \lambda_{i} \ldots\right) \text { (quantitative mapping), which }
\end{aligned}
$$
can also be expressed as:

$$
\Psi_{i}: f_{i} \rightarrow I F \quad \text { (condition) THEN (operation), }
$$

(qualitative mapping).

Decision-making is the key of character recognition, and it is also a decision-making method based on probability. It is based on similarity relationship. And the qualitative mapping relationship of the "show of hands".It is a quantitative mapping relation of distance function.

Decision model set $\Psi$ is composed of all decision models, and different decision models correspond to different identification environments.Multimodal Recognition $\Psi$ - --- Change the recognition method of recognition strategy, according to different situations, in the case of intelligent recognition.

Observational feature identification------The process of recognizing an object under what characteristic state and classifying it into a pattern category is the process of making use of decision mode.

\subsection{Condition 6:}

Use IF... THEN... Production rule description and reasoning are based on the result of feature identification. This is a simulation of human (expert) decision-making process. That is the rule set $\Omega$ of Heurestics and Intuition.

In summary, the information processing process of quadratic mapping relation is called human-simulated intelligent recognition theory, which is to solve the recognition task.

$$
\Omega_{1} \Phi \rightarrow \psi, \quad \Omega 1=\{\mathrm{y} 11, \mathrm{y} 12, \ldots, \mathrm{y} 1 \mathrm{r}\} \quad \text { (7) a }
$$

In the above formula: yli: IF $\phi_{i}$ THEN $\psi_{i}$ (qualitative mapping)

$$
\Psi_{1}: \quad R_{1} \stackrel{F_{1}}{\longrightarrow} U_{1}, \quad \Psi_{1}=\left\{\Psi_{11}, \Psi_{12}, \ldots, \Psi_{1 r}\right\} \quad \text { (7) b }
$$

$\Psi_{i}$ : Mapping relations can be qualitative or quantitative.

The above formula is simplified as two triple - order elements.

Let IC1 be an intelligent control process, thus:

$$
\mathrm{IC} 1=\left\langle\Phi_{1}, \Psi_{1}, \Omega_{1}\right\rangle, \Psi_{1}=\left\langle R_{1}, U_{1}, F_{1}\right\rangle
$$




\section{EXPERT OBSERVER -- DESIGN PROCESS}

Now in this paper, the structure of the expert system is simplified, a smart observer is formed by using rule set, knowledge base and database. Also known as the expert observer (EO).

In order to obtain the adaptive and flexible observation process and to satisfy the reliability and realtime performance of the motion system, we design an expert observer.Its basic structure diagram is as follows: Fig. 1:

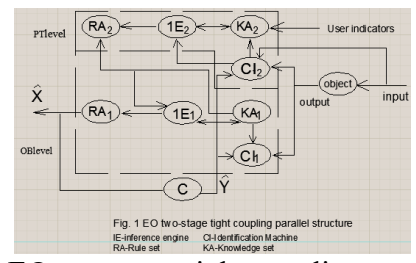

Figure 1. EO two-stage tight coupling parallel strcture

As can be seen from the above figure, the expert observer (EO) is composed of two tight coupling levels, namely parameter correction (PT) and observation level (OB), and the above two levels are running in parallel. The problem of real-time observation is corresponded to OB by observation level (OB), and the parameter correction problem is corresponded to $\mathrm{OB}$ observation mode by PT. Parameter correction (PT) and observation level (OB) have (RA) rule set, (KA) knowledge set, (CI) feature recognition machine, (IE) reasoning machine.

The first step is to identify characteristics. That is to carry out necessary detection, data processing and filtering on the dynamic error information of the observation process. The second step is to feed the characteristic quantity into the (EO) expert observer, which can reflect the characteristics of the observation process. On the basis of the process of knowledge set and the feature information of prior knowledge, the knowledge set can be supplemented or modified while providing the information needed to the reasoning machine. In order to quickly realize the function of selfcorrection and self-adaptive observation, the reasoning machine is used for reasoning, judgment and decisionmaking of appropriate observation mode and parameter correction strategy

\subsection{Characteristic model -- structure and identification}

The knowledge set established in this paper is a quantitative and qualitative combination of the dynamic error characteristic model and the observation model set, and the mapping relationship of the response.In the process of feature identification and EO design, it is the first step to construct the feature model of the observation system. The characteristic model of the observer system is composed of the main characteristic information of the motion state of the observer system.

\subsubsection{Condition 1:}

Expert observer (EO) levels of PT and OB the characteristics of the model $\Phi$, in terms of combined quantitative and qualitative, characteristics of system is described. The characteristic model $\Phi$, is a division of system dynamic information In space. It is divided according to the requirements of the state reconstruction and performance index of the observation object. By various divided into every area respectively to represent a kind of characteristics of the system state $\varphi \mathrm{I}$, characteristics of the status of all the collections are all levels of the characteristics of the model. As follows:

$\Phi=\{\varphi 1, \varphi 2, \ldots ., \varphi n\}, \varphi \mathrm{i} \in \operatorname{In} \quad$ (1)

By similar model in different group, in EO information space, composition characteristics of the model $\Phi$. The feature states of the feature model are described by the combination of feature elements.

The following represents the set of feature primitives:

$\mathrm{Q}=\{\mathrm{q} 1, \mathrm{q} 2, \cdots \mathrm{q} 3\}$

The error information space is set as:

q 1 : $\mid$ e $\mid<\delta 1$, q 2 : $|\dot{\text { e }}|<\delta 2$, q $3: \mid$ e $\mid<\beta 1$,

q $4:|\dot{\mathrm{e}}|>\beta 2$, q 5: ei- $1 \cdot \mathrm{ei} \geqslant 0$,

q6: $\mid$ ei-1/ei $\mid \geqslant \alpha 1$, q7 : ei- èi $\geqslant 0$, q8 : $\mid \dot{\mathrm{e}} / \mathrm{e}$ $>\alpha 2 \quad \cdots$ 。

In the upper formula, the threshold is $\alpha 1, \alpha 2, \delta 1$, $\delta 2, \beta 1, \beta 2$,

èi ------ Error rate at time I;

ei ------ Error value at time I;

The relationship between feature model and characteristic element $\mathrm{Q}$ is as follows:

$\Phi=\mathrm{P} \odot \mathrm{Q}$

$\Phi$------ $\mathrm{n}$ dimensional vector

Q ------ m dimensional vector

$\odot$------ The multiplicative relation of "and" matrices.

Pij ------ Take -1, 0, and 1 values to indicate that the inverse, zero and positive are taken.

$\mathrm{P}=[\mathrm{Pij}] \mathrm{n} \times \mathrm{m}$------ It is the relation matrix of $\mathrm{n} \times \mathrm{m}$ order.

\subsection{In the aspect of multi-observation mode ---- - Access and knowledge representation}

Multi - observation mode is the key of EO design. According to the expert's experience and knowledge, the piecewise approximation linearization mode is processed and reasonably simplified in the process of observing the object's motion state. The observation mode of the observer system is expressed in the set of all modes.

\subsubsection{Condition 2:}

The input information $U$ and the feature recognition memory information $\mathrm{R}$ of the observer at each stage constitute the state observation mode and the parameter set. Set of some quantitative or qualitative mapping relation $\mathrm{F}$ between parameter and state output information $\mathrm{X}$, as shown below:

$$
\begin{aligned}
& \Psi=\{\psi 1, \quad \psi 2, \cdots, \quad \psi p\} \\
& \psi \text { i ---- fi } \rightarrow \text { IF (conditions) }
\end{aligned}
$$

THEN(operation) (qualitative mapping) 
$\psi$ i ----- $\mathrm{Xi}=\mathrm{fi} \quad(\mathrm{U}, \mathrm{R}) \quad$ ( Quantitative projection)

Multimode observational reasoning decision --------It is obtained by changing the observation mode of the strategy.

Observation model set $\Psi$-------- each observation model is made up of some pattern primitives, is a kind of knowledge representation by EO experts prior knowledge to decide. As follows:

$\mathrm{A}=\{\mathrm{a} 1, \mathrm{a} 2, \cdots, \mathrm{ar}\}$

The relationship between observation mode elements and observation patterns is as follows:

$$
\Psi: \mathrm{X}=\mathrm{L} \cdot \mathrm{A}
$$

A ------ Schema primitive vector

L ----- Relation matrix

X ------ State output (selected elements are -1, 0, 1).

Commonly used observation pattern elements: state vector linearization approximate formula.The state vector physical formula and the open-loop observation primitive.

Empirical formula ------ (for example $\mathrm{Xi}=\mathrm{Xi}-1$ ( keep), Xi=Xi-1+k $\sum_{i=1}^{t} e_{i}$ Etc. ),

$\mathrm{K}$------ Error and weighted coefficients.

It is based on classical observer theory.

\subsection{Observation strategy ------ Decision making and reasoning}

EO design ------ the purpose is: data-driven rule reasoning based on feature recognition memory, on-line correction of observation strategy, observer parameters, in order to adapt to the changes of the observation process, flexible, reliable and accurate multi-mode observation strategy.

EO design ------ based on the mechanism of observing human activities (experts).

\subsubsection{Condition 3:}

The expert observer (EO) is an information processing process with quadratic mapping relation to solve the state reconstruction problem.

The following rules are used to represent: IF. . . THEN. . . , Can be written as:

- $\Phi-$ (mapping) $\rightarrow \Psi$

IF $\varphi$ i THEN $\psi$ i (Heuristic reasoning qualitative mapping)

- fi (U, R) - (mapping) $\rightarrow X$

IF fi ( U, R) THEN xi (Qualitative mapping)

$\mathrm{xi}=\mathrm{fi}(\mathrm{U}, \mathrm{R}) \quad$ (Quantitative mapping)

Special attention is paid to the fact that the necessary condition for establishing knowledge sets with two mapping relations is full mapping relation. If this necessary condition is not satisfied, then the appropriate state observations can not be measured, so that the target can not be searched from the reasoning mechanism. Therefore:
IF (conditions are not met) THEN (the operation is $\mathrm{xi}$ $=\mathrm{xi}-1$ ), that is: the last rule in knowledge concentration should be the default value.

In summary, on-line feature identification is based on the dynamic characteristics of the observed objects, multi-mode observation is based on the classical observer design theory, and the prior knowledge of feature model establishment is needed.

Expert observer design method is composed of computer technology knowledge, artificial intelligence, observer design theory.

\section{CONCLUSION}

In this paper, the mechanism of intelligent image recognition is studied, designed, and the experimental results are presented. In short, the following expressions:

- The main objective of the study is to combine qualitative and quantitative expert knowledge model, which is based on the dynamic feature model of feature identification. Instead of using the object corresponding to the mathematical model of the observer, the results are obtained.

- The research is based on two level tightly coupled information processing and multiple observation mode reasoning decision-making. The system has the ability of on-line self-tuning and self adaptation.

- Experiments have proved that the advantage of expert observer becomes more and more obvious with the difficulty of object observation

- The expert observer with simple structure, good real-time performance, strong modularity and strong engineering practicability can be obtained by using the generated data-driven reasoning rules.

- More in-depth research is needed for those with high design difficulty.

\section{References}

1. Xu Liyan, Liu Fuchang, Cao Guo, Sun Quansen, Xia Deshen. Color image segmentation method based on edge flow and region merging. J. Optoelectronics. Laser, 10(2011)

2. WuXiaoHong,LuoDaiSheng.Integration of core image of the edge flow and normalized cut segmentation. J. Journal of Optoelectronics.Laser; 03(2010)

3. HeYue,ShenXuanJing,CengZheng. One kind of effective suppression noise fuzzy $\mathrm{C}$ average value image division algorithm. J. Computer Engineering and Applications, 29(2011)

4. ZhangYiXing,WangXia,FangMing,LiXiaoDong,Lin gFeng. Based on spatial information possible fuzzy $\mathrm{C}$ average value cluster remote sensing image division. J. Journal of Computer Applications, 11 (2011)

5. ChenZiYang;WangBaoPing. One kind based on three dimensional histogram and RFKCN image division method. J. Chinese Journal of Computers, 08(2011) 